\title{
ALTERAÇÕES DO SISTEMA CARDIOPULMONAR DE PACIENTES CIRRÓTICOS
}

\author{
CHANGES IN THE CARDIOPULMONARY SYSTEM OFCIRRHOTIC PATIENTS
}

Artigo Original

CAMBIOS ENEL SISTEMA CARDIOPULMONARDE PACIENTES CIRRÓTICOS

Rodrigo Casales da Silva Vieira' (Fisioterapeuta)

Mario Reis Álvares-da-Silva² (Médico)

Álvaro Reischak de Oliveira' (Profissional de Educação Física) Julia da Silveira Gross ${ }^{1}$ (Profissional de Educação Física) Renata Lopes Kruger' (Profissional de Educação Física) Adriane Dal Bosco 1,3 (Fisioterapeuta)

Norma Anair Possa Marroni2,4 (Bióloga)

Luiz Alberto Forgiarini Junior ${ }^{3}$ (Fisioterapeuta)

Alexandre Simões Dias 1,2,5

(Fisioterapeuta)

1. Universidade Federal do Rio Grande do Sul (UFRGS), Porto Alegre, RS, Brasil.

2. Universidade Federal do Rio Grande do Sul, Hospital de Clínicas de Porto Alegre (HCPA), Porto Alegre, RS, Brasil.

3. Centro Universitário Metodista (IPA), Porto Alegre, RS, Brasil. 4. Universidade Luterana do Brasil (ULBRA), Canoas, RS, Brasil. 5. Hospital de Clínicas de Porto Alegre (HCPA), Serviço de Fisioterapia, Porto Alegre, RS, Brasil.

\section{Correspondência:}

Rodrigo Casales da Silva Vieira Universidade Federal do

Rio Grande do Sul.

Rua General João Telles, 347, complemento 201, Bom Fim, Porto Alegre, RS, Brasil. 90035-121. rodrigocasales@bol.com.br rodrigocasales@hotmail.com

\section{RESUMO}

Introdução: A cirrose gera alterações nas trocas gasosas e a desnutrição proteico-calórica em pacientes hepatopatas. Objetivo: Avaliar e comparar as variáveis cardiopulmonares, a força do aperto de mão (FAM) e a composição corporal entre pacientes cirróticos pelo vírus da hepatite $C$ e indivíduos saudáveis, e correlacionar o consumo máximo de oxigênio $\left(\mathrm{VO}_{2 \mathrm{MAX}}\right)$ com a FAM. Métodos: Esta pesquisa caracteriza-se como estudo de caso-controle composto por 36 participantes (18 pacientes cirróticos com HCV e 18 indivíduos hígidos) de ambos os sexos, maiores de 18 anos. A força de preensão palmar foi mensurada por dinamometria com dinamômetro mecânico de empunhadura com alça ajustável. As variáveis ventilatórias foram avaliadas por ergoespirometria com teste de carga progressiva em cicloergômetro. A composição corporal foi mensurada por um técnico em cineantropometria nível II. Foram utilizados os testes $t$ independente e Mann-Whitney para comparação entre os grupos e a correlação de Spearman para associação entre as variáveis. Resultados: Foram encontradas diferenças no consumo máximo de oxigênio 16,20 (11,60-18,55), mediana e intervalo interquartil x 19,90 $(16,27-26,85)$, ventilação 45,40 (36,45-54,20) x 63,40 (50,40-78,00), produção de dióxido de carbono 785,88 $(655,81-963,14) \times$ 988,04 (826,93-1546,21), frequência cardíaca máxima $(127,66 \pm 23,26$ média e \pm DP) $\times(146,29 \pm 23,31)$, primeiro limiar ventilatório $(10,700 \pm 3,19) \times(14,912 \pm 4,45)$ e segundo limiar ventilatório $(14,16 \pm 4,48) \times$ $(18,25 \pm 5,54)$ entre cirróticos e controles, respectivamente. Encontramos correlação positiva moderada entre o consumo máximo de oxigênio e a força do aperto de mão $(r=0,474, p=0,047)$. Conclusão: Existem alterações nas variáveis cardiopulmonares e há associação entre o $\mathrm{VO}_{2 \mathrm{MAX}}$ e a FAM em pacientes cirróticos pelo vírus da hepatite $C$.

Descritores: força da mão; cirrose hepática; consumo de oxigênio.

\section{ABSTRACT}

Introduction: Cirrhosis causes changes in gas exchange and protein-calorie malnutrition in patients with liver disease. Objective: To evaluate and compare cardiopulmonary variables, handgrip strength (HGS) and body composition between cirrhotic patients with hepatitis C virus and healthy individuals, and to correlate maximal oxygen uptake $\left(\mathrm{VO}_{2 \mathrm{MAX}}\right)$ with HGS. Methods: This survey is characterized as a case-control study composed of 36 participants (18 cirrhotic patients with HCV and 18 healthy individuals) of both sexes, older than 18 years. The palmar grip strength was measured by dynamometry using a mechanical handle dynamometer with adjustable handle. The ventilatory variables were evaluated by ergospirometry with a progressive load test on a cycloergometer. The body composition was measured by a level II cineanthropometry technician. Independent t test and Mann-Whitney test were used for comparison between groups and Spearman correlation for association between variables. Results: There were differences in maximal oxygen uptake 16.20 (11.60-18.55), median and interquartile range x 19.90 (16.27-26.85), ventilation 45.40 (36.45-54.20) x 63.40 (50.40-78.00), carbon dioxide production 785.88 (655.81-963.14) $\times 988.04$ (826.93-1546.21), maximum heart rate (127.66 \pm 23.26 , mean and \pm SD) $\times(146.29 \pm 23.31)$, first ventilatory threshold $(10.700 \pm 3.19) \times(14.912 \pm 4.45)$ and second ventilatory threshold $(14.16 \pm 4.48) \times(18.25 \pm 5.54)$ between cirrhotic patients and controls, respectively. We found a moderate positive correlation between maximal oxygen uptake and handgrip strength $(r=0.474, p=0.047)$ Conclusion: There are changes in cardiopulmonary variables and there is an association between $V_{2 M A x}$ and HGS in cirrhotic patients with hepatitis C virus.

Keywords: hand strength; liver cirrhosis; oxygen consumption.

\section{RESUMEN}

Introducción: La cirrosis genera cambios en los intercambios gaseosos y la desnutrición proteico-calórica en pacientes con enfermedad hepática. Objetivo: Evaluar y comparar las variables cardiopulmonares, la fuerza de prensión manual (FPM) y la composición corporal entre pacientes cirróticos por el virus de la hepatitis Cy sujetos sanos, y correlacionar el consumo máximo de oxígeno $\left(V_{2}{ }_{2 M A x}\right)$ con la FPM. Métodos: Esta investigación se caracteriza como estudio caso-control compuesto por 36 participantes (18 pacientes cirróticos con VHC y 18 individuos sanos) de ambos sexos, mayores de 18 años. La fuerza de agarre palmar se midió mediante dinamometría con dinamómetro mecánico con mango ajustable. Las variables ventilatorias se evaluaron mediante 
ergoespirometría con prueba de carga progresiva en un cicloergómetro. La composición corporal fue medida por un técnico en cineantropometría de nivel II. Se utilizaron las pruebas t independiente y Mann-Whitney para la comparación entre los grupos y la correlación de Spearman para la asociación entre las variables. Resultados: Se encontraron diferencias en el consumo máximo de oxígeno 16,20 (11,60-18,55), mediana y rango intercuartílico x 19,90 (16,27-26,85), ventilación 45,40 (36,45-54,20) x 63,40 (50,40-78,00), producción de dióxido de carbono $785,88(655,81-963,14) \times 988,04(826,93-1546,21)$, frecuencia cardiaca máxima $(127,66 \pm 23,26$, media y $\pm D E) \times$ $(146,29 \pm 23,31)$, primer umbral ventilatorio $(10,700 \pm 3,19) \times(14,912 \pm 4,45)$ y segundo umbral ventilatorio $(14,16$ $\pm 4,48) \times(18,25 \pm 5,54)$ entre los cirróticos y controles, respectivamente. Encontramos una correlación positiva moderada entre el consumo máximo de oxígeno y la fuerza de prensión manual ( $r=0,474, p=0,047)$. Conclusión: Existen cambios en las variables cardiopulmonares y hay asociación entre el $V_{2}{ }_{2 M A x}$ y FPM en pacientes cirróticos por el virus de la hepatitis $C$.

Descriptores: fuerza de la mano; cirrosis hepática; consumo de oxígeno.

\section{INTRODUÇÃO}

A cirrose hepática é o estádio final comum de uma série de processos patológicos hepáticos de diversas causas como a infecção crônica pelo Vírus da Hepatite $C^{1}$.

Uma das complicações da cirrose é a desnutrição protéico-calórica, um achado frequente em pacientes com hepatopatia avançada ${ }^{2,3}$. Atualmente, existem alguns métodos de avaliação nutricional como a avaliação antropométrica, que abrange a mensuração de medidas como peso, altura, índice de massa corporal (IMC) e dobras cutâneas ${ }^{4}$. A antropometria permite que se avalie a composição corporal que é a capacidade do ser humano em realizar qualquer tipo de esforço tendo relação com a maior ou a menor presença dos seus tecidos corporais fundamentais ${ }^{5}$.

Outras complicações oriundas da cirrose são a diminuição da pressão parcial de oxigênio arterial $\left(\mathrm{PaO}_{2}\right)$ e na relação de oxigênio com a hemoglobina $\left(\mathrm{SatO}_{2} / \mathrm{Hb}\right)$ e o aumento na pressão parcial de gás carbônico $\left(\mathrm{PaCO}_{2}\right)$ que ocorrem devido as alterações nas trocas gasosas entre o tecido pulmonar e o tecido sanguíneo ${ }^{6}$.

A associação entre doença hepática e anormalidades e sintomas pulmonares são comuns em pacientes hepatopatas crônicos e têm sido reconhecidas há mais de 100 anos. Entretanto, somente nos últimos quinze anos alterações vasculares pulmonares específicas associadas com a presença de doença hepática têm sido submetidas a maiores investigações.

A doença hepática associada às alterações pulmonares causa um grande impacto nutricional, pois o fígado realiza inúmeras reações bioquímicas, como produção, modificação e utilização de nutrientes e substâncias metabolicamente importantes para o organismo.

O objetivo do estudo foi avaliar e comparar as variáveis ventilatórias, a força do aperto de mão e a composição corporal entre pacientes cirróticos pelo vírus da hepatite $C$ e indivíduos saudáveis e correlacionar a força do aperto de mão com o consumo máximo de oxigênio em pacientes cirróticos.

\section{MATERIAIS E MÉTODOS}

O presente estudo caracteriza-se como um estudo de caso-controle em que foram avaliados 36 participantes (18 pacientes cirróticos e 18 indivíduos saudáveis). Os grupos foram pareados através do sexo e da idade.

Tamanho da amostra: Foi obtido a partir do estudo de Galant et al. ${ }^{9}$, que investigou a capacidade funcional de indivíduos com cirrose através do teste de caminhada de seis minutos.

Critérios de inclusão: Os pacientes deveriam ter o diagnóstico clínico de cirrose hepática compensada com etiologia viral C (VHC) e estar em acompanhamento clínico no Ambulatório de Hepatites Crônicas do Serviço de Gastroenterologia do Hospital de Clínicas de Porto Alegre - HCPA.
Critérios de exclusão: Foram excluídos indivíduos que apresentassem outras causas de doença hepática, varizes esofágicas grau 3 e 4, anemia, coinfecção com outros vírus, insuficiência renal crônica, dispnéia em repouso e ou que fossem usuários de drogas lícitas e ou ilícitas.

Perdas amostrais: Indivíduos de ambos os grupos que apresentassem alterações nos sinais vitais antes ou durante a realização dos testes propostos.

Procedimentos éticos: Este estudo foi aprovado pelo comitê de ética e pesquisa do HCPA e todos os participantes assinaram o termo de consentimento livre e eslcarecido antes de serem avaliados.

Avaliações: Os participantes selecionados foram submetidos à avaliação da composição corporal, da força do aperto de mão e do teste de esforço máximo cardiopulmonar.

Composição corporal: Foi mensurada por um mesmo técnico em cineantropometria nível II da International Society for the Advancement of Kineanthropometry (ISAK), utilizando procedimentos estabelecidos pela própria instituição ${ }^{10}$. As marcações dos locais e a técnica de tomada das dobras cutâneas seguiram os padrões da Sociedade Internacional para o Avanço da Cineantropometria (ISAK). As mensurações de estatura, massa corporal, comprimentos, diâmetros, perímetros e dobras cutâneas permitiram que a massa total fosse fracionada em cinco componentes (massa muscular, massa adiposa, massa residual, massa óssea e massa epitelial), o que proporcionou uma descrição e comparação detalhada da composição corporal dos participantes ${ }^{10}$.

Força do aperto de mão (FAM): Foi verificada através do dinamômetro mecânico de empunhadura com alça ajustável do tipo Baseline Smedley Spring-Type Hand Dynamometer (New York, USA). O valor obtido foi a média das três aferições da mão não dominante, as quais não deveriam diferir 10\% entre as mensurações ${ }^{11}$.

Teste de esforço máximo cardiopulmonar: Foi mensurado através de um sistema de ergoespirometria de circuito aberto por analisador de gases (MGC, modelo CPX/D). Os testes de carga progressiva, em cicloergômetro (The Bike, Cibex, USA), foram realizados segundo protocolo em rampa. A intensidade inicial estabelecida foi de $25 \mathrm{~W}$, com aumento de $25 \mathrm{~W}$ a cada dois minutos (25W. $\left.\mathrm{min}^{-2}\right)$, mantendo uma cadência entre 70 e 80 rotações por minuto (rpm) ${ }^{12}$. Os participantes informaram sobre a taxa de percepção subjetiva de esforço a cada aumento de intensidade e foram verbalmente estimulados para que realizassem esforço máximo durante o teste que teve duração entre 8-12 minutos de acordo com as recomendações do American College of Sports Medicine (ACSM) e foi encerrado quando os participantes atingiram um dos seguintes critérios: pico e/ou platô no consumo de oxigênio; frequência cardíaca $\geq$ predita para idade; Valor de taxa 
de troca respiratória $>1,15$; percepção subjetiva de esforço $>18$ ou quando o participante voluntariamente interrompeu o teste $\mathrm{e}^{13,14}$. No dia do teste os indivíduos foram instruídos a estarem alimentados, porém com intervalo de 2 horas da última refeição para evitar desconfortos ou náuseas.

Foram verificadas através do teste cardiopulmonar as seguintes variáveis ventilatórias: consumo máximo de oxigênio $\left(\mathrm{VO}_{2 \max }\right)$, produção de dióxido de carbono $\left(\mathrm{VCO}_{2}\right)$, limiares ventilatorios 1 e 2 (LV1absoluto - LV2 absoluto), ventilação (VE) e frequência cardiaca máxima ( $\left.F C_{\max }\right)$.

A determinação dos limiares ventilatórios e do $\mathrm{VO}_{2 \text { pico }}$ obedeceu os seguintes critérios:

- $\quad$ LV foi considerado como a mínima carga em que VENO $\mathrm{NO}_{2}$ apresentou um aumento sistemático sem um aumento concomitante de $\mathrm{VE} / \mathrm{NCO}_{2}$;

- $\quad \mathrm{LV}_{2}$ foi considerado como a mínima carga em que VENO $\mathrm{NO}_{2}$ apresentou um aumento concomitante com VE/ $\mathrm{NCO}_{2}$;

- Caso nenhum dos critérios acima estabelecesse o $\mathrm{LV}_{2}$, um aumento não linear do RER foi utilizado;

- $\mathrm{O} \mathrm{VO}_{2 \max }$ foi considerado como a intensidade mínima em que os valores de $\mathrm{VO}_{2}$ atingissem um platô e/ou pico. Um platô foi considerado como uma variação inferior a $1,5 \mathrm{ml} . \mathrm{kg}^{-1} \cdot \mathrm{mim}^{-1}$ mesmo com incrementos subsequentes da intensidade do exercício.

\section{Análise estatística}

Foi desenvolvida no software SPSS 18.0, adotando-se um nível de significância de 5\% ( $p<0,05)$ e poder de $80 \%$. Para testar a distribuição dos dados foi utilizado o teste de normalidade Shapiro Wilk. As variáveis qualitativas foram descritas em proporção e porcentagem respectivamente. Para comparação entre os grupos foi utilizado o teste Qui-quadrado. As variáveis quantitativas foram descritas em media e desvio padrão e mediana e intervalo interquartil. A comparação entre os dois grupos foi realizada pelos Testes $t$ - Independente e Mann-Withney. Para correlação das variáveis foi utilizado o teste de Correlação de Spearman.

\section{RESULTADOS}

As características antropométricas dos participantes estão descritas na Tabela 1. Observaram-se resultados semelhantes nos dois grupos quanto à idade, gênero, peso, altura e índice de massa corporal (IMC) não havendo diferenças estatísticas entre os participantes.

Foram encontradas diferenças entre os grupos no teste ergoespirométrico para o $\mathrm{VO}_{2 \text { max }}, \mathrm{VE}, \mathrm{VCO}_{2}, \mathrm{LV} 1$ absoluto, LV2 absoluto e $\mathrm{FC}_{\text {MAX }}$. Os dados estão descritos na Tabela 2.

Não foram encontradas diferenças entre os grupos para a composição corporal nos seus cinco componentes (massa adiposa, massa muscular, massa residual, massa óssea e massa epitelial). Os dados estão expressos na Tabela 3.

Quanto à FAM não houve diferenças estatísticas entre cirróticos $(1,89 \pm 0,90$ média e $\pm D P)$ e controles $(2,46 \pm 0,116),(p=1,183)$. Contudo, obtivemos uma correlação positiva moderada entre o VO2max e a FAM $(r=0,474, p=0,047)$, como pode ser visto na Figura 1 .

Tabela 1. Características antropométricas dos pacientes cirróticos e dos indivíduos hígidos.

\begin{tabular}{c|c|c|c}
\hline Características & $\begin{array}{c}\text { Cirróticos } \\
(\mathbf{n = 1 8 )}\end{array}$ & $\begin{array}{c}\text { Controles } \\
(\mathbf{n = 1 8 )}\end{array}$ & $\mathbf{p}$ \\
\hline Idade (anos) & $55,61 \pm 8,31$ & $55,22 \pm 8,85$ & 0,893 \\
\hline Genêro (M/F) & $8(44,4 \%) / 10(55,6 \%)$ & $8(44,4 \%) / 10(55,6 \%)$ & 1,000 \\
\hline Peso $(\mathrm{Kg})$ & $66,50(57,62-73,10)$ & $70,90(63,85-75,62)$ & 0,229 \\
\hline Altura $(\mathrm{cm})$ & $157,45(153,75-166,68)$ & $163,30(154,75-169,63)$ & 0,261 \\
\hline IMC $\left(\mathrm{Kg} \backslash \mathrm{cm}^{2}\right)$ & $26,04(21,98-28,77)$ & $25,32(24,13-26,70)$ & 0,975 \\
\hline
\end{tabular}

M/F - masculino/feminino: $\mathrm{Kg}$ - kilograma $\mathrm{cm}$ - centímetros: $\mathrm{Kg} / \mathrm{cm}^{2}-$ quilograma por centímetro quadrado; dados descritos em média e desvio padrăo; proporção e porcentagem e mediana e intervalo interquarti $\left(P_{25}-P_{75}\right) ; p$ - nível de significância $(\leq 0,05)$.
Tabela 2. Diferença entre os grupos (cirrótico e controle) para os dados ergoespirométricos

\begin{tabular}{c|c|c|c}
\hline Variáveis & $\begin{array}{c}\text { Cirróticos } \\
(\mathbf{n}=\mathbf{1 8})\end{array}$ & $\begin{array}{c}\text { Controles } \\
(\mathbf{n = 1 8 )}\end{array}$ & $\mathbf{p}$ \\
\hline $\begin{array}{c}\mathrm{VO}_{2 \mathrm{max}} \\
\left(\mathrm{ml} / \mathrm{Kg}^{-1} / \mathrm{min}^{-1}\right)\end{array}$ & $16,20(11,60-18,55)$ & $19,90(16,27-26,85)$ & $0,007^{*}$ \\
\hline $\mathrm{VE}(\mathrm{L} / \mathrm{min})$ & $45,40(36,45-54,20)$ & $63,40(50,40-78,00)$ & $0,004^{*}$ \\
\hline $\mathrm{VCO}(\mathrm{ml} / \mathrm{min})$ & $785,88(655,81-963,14)$ & $988,04(826,93-1546,21)$ & $0,010^{*}$ \\
\hline $\begin{array}{c}\mathrm{L} 1 \mathrm{absoluto} \\
\left(\mathrm{ml} / \mathrm{Kg}^{-1} / \mathrm{min}^{-1}\right)\end{array}$ & $10,70 \pm 3,19$ & $14,91 \pm 4,45$ & $0,004^{*}$ \\
\hline $\begin{array}{c}\mathrm{L} 2 \mathrm{absolutO}^{-1} \\
\left(\mathrm{ml} / \mathrm{Kg}^{-1} / \mathrm{min}^{-1}\right)\end{array}$ & $14,16 \pm 4,48$ & $18,25 \pm 5,54$ & $0,024^{*}$ \\
\hline $\mathrm{FC}_{\mathrm{Max}}(\mathrm{bpm})$ & $127,66 \pm 23,26$ & $146,29 \pm 23,31$ & $0,031^{*}$ \\
\hline
\end{tabular}

Consumo máximo de oxigênio $\left(\mathrm{VO}_{2 \mathrm{max}}\right)$; produção de dióxido de carbono $\left(\mathrm{VCO}_{2}\right)$; limiares ventilatorios 1 e 2 (LV1absoluto - LV2 absoluto); ventilação (VE); frequência cardíaca máxima $\left(F C_{m a x}\right) ; \mathrm{ml} / \mathrm{Kg} / \mathrm{min}$ - mililitro por quilograma por minuto: L/min - litro por minuto bpm - batimentos por minuto dados descritos em medina

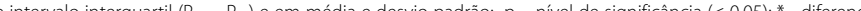
estatística entre os grupos.

Tabela 3. Comparação entre os grupos para composição corporal (massa adiposa massa muscular, massa residual, massa óssea e massa epitelial).

\begin{tabular}{c|c|c|c}
\hline Variáveis & $\begin{array}{c}\text { Cirróticos } \\
(\mathbf{n = 1 8 )}\end{array}$ & $\begin{array}{c}\text { Controles } \\
(\mathbf{n}=\mathbf{1 8})\end{array}$ & $\mathbf{p}$ \\
\hline massa adiposa (\%) & $37,75 \pm 9,52$ & $37,31 \pm 5,32$ & 0,867 \\
\hline massa muscular (\%) & $34,14 \pm 8,94$ & $36,59 \pm 5,33$ & 0,324 \\
\hline massa residual (\%) & $10,96 \pm 2,32$ & $10,75 \pm 1,21$ & 0,728 \\
\hline massa óssea (\%) & $9,85(9,100-11,075)$ & $10,700(9,950-11,300)$ & 0,342 \\
\hline massa epitelial (\%) & $5,00(4,47-5,47)$ & $4,90(4,70-5,12)$ & 0,623 \\
\hline
\end{tabular}

$\%)$ - porcentagem; dados descritos em média e desvio padrão e mediana e intervalo interquartil $\left(P_{25}-P_{75}\right)$ - nível de significancia $(\leq 0,05$.

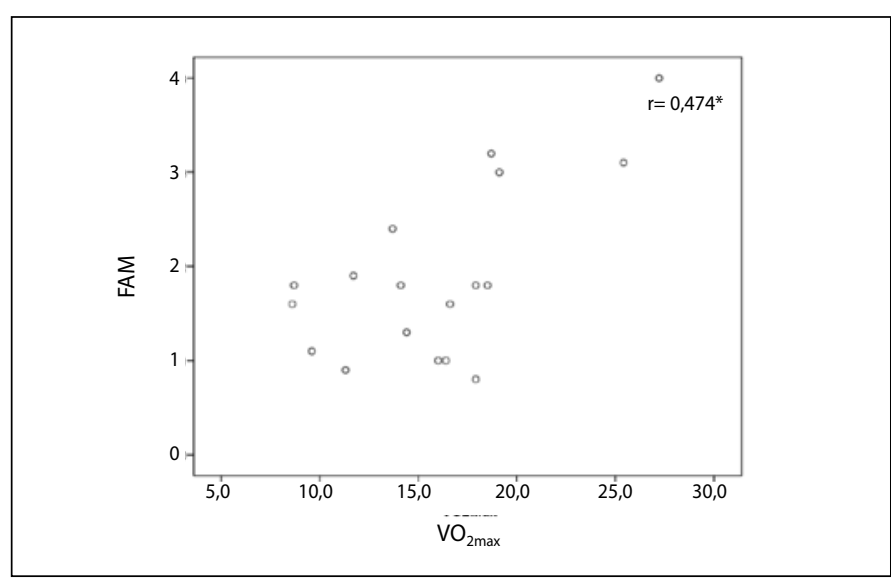

Figura 1. Relação entre a força do aperto de mão (FAM) e o consumo máximo de oxigênio $\left(\mathrm{VO}_{2 \max }\right)$

\section{DISCUSSÃO}

Os principais resultados do presente estudo foram que as variáveis ergoespirométricas $\mathrm{VO}_{2 \text { max }}, \mathrm{VE}, \mathrm{VCO}$, LV1absoluto, $\mathrm{LV} 2$ absoluto e $\mathrm{FC}_{\mathrm{MAX}}$ apresentaram-se alteradas no grupo cirrótico em relação ao grupo controle (Tabela 2). Jones et al. ${ }^{15}$ descreveram em sua revisão sistemática que a maioria dos estudos que avaliaram a capacidade aeróbia em cirróticos, sob o teste cardiopulmonar de esforço máximo, mensuraram, apenas, o $\mathrm{VO}_{2 \text { pico }}$ verificando importante redução nessa variável. Um estudo verificou que oito pacientes com cirrose compensada apresentaram uma redução de $82 \%$ no VO $_{2 \text { pico, }}$ considerando-se o sexo e a idade. Outro estudo encontrou uma redução de $60 \%$ a $78 \%$ no $\mathrm{VO}_{2 \text { pico }}$ em comparação a indivíduos saudáveis. Poucos estudos, no entanto, avaliaram o metabolismo anaeróbico durante o exercício. Todos eles sugerem que o metabolismo anaeróbico ocorra mais cedo nos cirróticos quando comparado à indivíduos saudáveis indo ao encontro dos resultados encontrados em nosso estudo ${ }^{15}$. 
A força do aperto de mão e a composição corporal não foram diferentes estatisticamente entre os grupos (Tabela 3). Contudo, os valores encontrados no grupo cirrótico para a FAM foram inferiores aos encontrados no grupo controle sugerindo que os cirróticos estavam piorando sua condição muscular e nutricional em virtude da patologia. A força do aperto de mão é útil nessa população, pois identifica os pacientes mais propensos a desenvolver complicações da cirrose, uma vez que reflete mudanças que ocorrem em músculos e ou grupos musculares mais nobres, como o diafragma ${ }^{11}$. Além disso, é o método que identifica os pacientes mais propensos à desnutrição. Nunes et al. ${ }^{16}$ avaliaram a força do aperto de mão em indivíduos com cirrose pelo vírus C classificados em seu grau de severidade como Child-Pilg A e concluíram que a FAM foi o método que mais identificou desnutrição nesses pacientes se comparado com outros métodos como a antropometria. Todavia, são necessários mais estudos que comprovem que o estado nutricional de pacientes com cirrose seja refletido pelo músculo ${ }^{11}$.

Em nosso estudo, associamos um método de avaliação nutricional (antropometria) com um método de avaliação funcional (FAM). Nunes et al. ${ }^{16}$ relataram que não há um método de avaliação nutricional considerado padrão áureo em pacientes cirróticos, sugerindo o uso combinado de métodos de avaliação nutricional associados com métodos funcionais na avaliação de indivíduos com cirrose. Além disso, a má nutrição energética proteica é um achado comum nos pacientes com cirrose hepática de etiologia etanólica, mas o valor prognóstico do metabolismo energético nos cirróticos de etiologia viral ainda não é bem estabelecido ${ }^{17}$.

A relação existente entre a força do aperto de mão e o $\mathrm{VO}_{2 \max } \mathrm{em}$ indivíduos cirróticos encontrada em nosso estudo (Figura 1) pode ser explicada pelas alterações encontradas na vasodilatação desses indivíduos interferindo na vasoconstrição arteriolar causando desvio do fluxo sanguíneo dos tecidos não ativos para os músculos esqueléticos em exercício, limitando a extração de oxigênio que é verificada em praticamente todos os pacientes com cirrose hepática cujo a magnitude desse prejuízo aumenta com a progressão da doença ${ }^{18,19}$.

O presente estudo teve como limitações a ausência de mensuração do tratamento medicamentoso para o VHC e sobre a presença de comorbidades como diabete mellitus tipo 2 e hipertensão arterial sistêmica, uma vez que o $\mathrm{VO}_{2 \text { Max }}$ possui associação com sexo, idade, presença de doenças e uso de medicações em pacientes hepatopatas ${ }^{20,21}$.

Sugere-se que, em futuros estudos, seja avaliado o sistema neuromuscular em conjunto com o sistema cardiorrespiratório, verificando-se - grau de ativação neuromuscular e a produção de força em pacientes cirróticos por vírus $\mathrm{C}$. Existem poucos dados sobre a capacidade de geração de força em cirróticos, os estudos existentes avaliam a força através da mensuração do torque isocinético e isométrico. Sabe-se que a força muscular nesses doentes apresenta-se reduzida em diferentes grupos musculares em relação a indivíduos saudáveis ${ }^{22}$.

\section{CONCLUSÃO}

Concluímos que existem alterações no consumo máximo de oxigênio, na produção de dióxido de carbono, na ventilação e nos limiares ventilatórios de cirróticos em relação a indivíduos saudáveis e que há uma associação entre a força do aperto de mão e o consumo máximo de oxigênio em pacientes com cirrose hepática pelo vírus da hepatite $C$.

\section{AGRADECIMENTOS}

Agradecemos à Coordenação de Aperfeiçoamento de Pessoal de Nível Superior (CAPES). Fundo de Incentivo a Pesquisa do Hospital de Clínicas de Porto Alegre (FIPE/HCPA).

Todos os autores declararam não haver qualquer potencial conflito de interesses referente a este artigo.

CONTRIBUIÇÕES DOS AUTORES: Cada autor contribuiu individual e significativamente para o desenvolvimento do manuscrito. RCSV (0000-0001-6758-327X)* foi o principal contribuinte para a redação do manuscrito. RCSV, MRAS (0000-0002-5001-246X)* e ASD (0000-0000-0609-4779)*, selecionaram os participantes do estudo. RCSV, JSG (0000-0002-7290-3466)* e RLK (0000-0001-7816-8127)* aplicaram os testes propostos no estudo. RCSV acompanhou os participantes do estudo em todos as suas etapas. RCSV e JSG realizaram a coleta de dados. RCSV, ASD, MRAS, ARO (0000-0003-4590-2991)* e LAFJ (0000-0002-6706-2703)* contribuíram significativamente na concepção e no desenho do manuscrito. RCSV, JSG, RLK e ASD contribuíram na revisão bibliográfica do estudo. RCSV, ASD, LAFJ, ADB (0000-0003-0907-8325)*, NAPM (000-0001-8623-1974)* contribuíram para a revisão crítica do manuscrito. RCSV, ASD, LAFJ, AB, NAPM e ARO contribuíram para a revisão e aprovação final do manuscrito. ${ }^{*} \mathrm{ORCID}$ (Open Researcher and Contributor ID).

\section{REFERÊNCIAS}

1. Schuppan D, Afdhal NH. Liver cirrhosis. Lancet. 2008;371(9615):838-51.

2. Peng S, Plank LD, McCall JL, Gillanders LK, Mcllroy K, Gane EJ. Body composition, muscle function, and energy expenditure in patients with liver cirrhosis: a comprehensive study. Am J Clin Nutr. 2007;85(5):1257-66.

3. Tsiaousi ET, Hatzitolios Al, Trygonis SK, Savopoulos CG. Malnutrition in end stage liver disease: recommendations and nutritional support. J Gastroenterol Hepatol. 2008;23(4):527-33.

4. Ritter L, Gazzola J. Nutritional evaluation of the cirrhotic patient: an objective, subjective or multicompartmental approach?. Arq Gastroenterol. 2006;43(1):66-70.

5. Ross WD, Kerr DA. Fraccionamiento de la masa corporal: un nuevo método para utilizar en nutrición clínica y medicina deportiva. Apunts Med Esport. 1993;18:175-87.

6. Marroni NP, Morgan-Martins MI, Porawski M. Radicais livres no processo saúde-doença: da bancada à clínica. Curitiba: CRV; 2012.

7. Rodríguez-Roisin R, Agustí AG, Roca J. The hepatopulmonary syndrome: new name, old complexities. Thorax. 1992;47(11):897-902.

8. Maio R, Dichi JB, Burini RC. Nutritional consequences of metabolic impairment of macronutrients in chronic liver disease. Arq Gastroenterol. 2000;37(1):52-7.

9. Galant LH, Ferrari R, Forgiarini LA Jr, Monteiro MB, Marroni CA, Dias AS. Relationship between MELD severity score and the distance walked and respiratory muscle strength in candidates for liver transplantation. Transplant Proc. 2010;42(5):1729-30.

10. Mafell-Jones M, Olds T, Stewart A, Carter L. International standards for anthropometric assessment. Potchefstroom, SA: ISAK; 2006

11. Álvares-da-silva MR, Silveira TR. Comparison between handgrip strength, subjective global assessment and prognostic nutritional index in assessing malnutrition and predicting clinical outcome in cirrhotic outpatients. Nutrition. 2005;21(2):113-7.
12. American College of Sports Medicine. Diretrizes do ACSM para os testes de esforço e sua prescrição. $7^{\text {a }}$ ed. Rio de Janeiro: Guanabara Koogan; 2007.

13. Wasserman K, Mcilroy MB. Detecting the threshold of anaerobic metabolism in cardiac patients during exercise. Am J Cardiol. 1964;14:844-52.

14. Dekerle J, Baron B, Dupont L, Vanvelcenaher J, Pelayo P. Maximal lactate steady state, respiratory compensation threshold and critical power. Eur J Appl Physiol. 2003;89(3-4):281-8.

15. Jones JC, Coombes JS, Macdonald GA. Exercise capacity and muscle strength in patients with cirrhosis. Liver Transpl. 2012;18(2):146-51.

16. Nunes FF, Fernandes SA, Bertolini CM, Rabito El, Gottschall CBA. Avaliação nutricional do paciente cirrótico: comparação entre diversos métodos. Sci Med. 2012;22(1):12-7.

17. Tajika M, Kato M, Mohri H, Miwa Y, Kato T, Ohnishi H, et al. Prognostic value of energy metabolism in patients with viral liver cirrhosis. Nutrition. 2002;18(3):229-34.

18. Epstein SK, Ciubotaru RL, Zilberberg MD, Kaplan LM, Jacoby C, Freeman R, et al. Analysis of impaired exercise capacity in patients with cirrhosis. Dig Dis Sci. 1998;43(8):1701-7.

19. Lemyze M, Dharancy S, Nevière R, Pruvot FR, Declerck N, Wallaert B. Aerobic capacity in patients with chronic liver disease: Very modest effect of liver transplantation. Presse Med. 2010;39(7-8):e174-81.

20. Arena R, Myers J, Guazzi M. The clinical importance of cardiopulmonary exercise testing and aerobic training in patients with heart failure. Braz J Phys Ther. 2008;12(2):75-87

21. Braga AM, Rondon MU, Negrão CE, Wajngarten M. Predictive value of ventilatory and metabolic variables for risk of death in patients with cardiac failure. Arq Bras Cardiol. 2006;86(6):451-8.

22. Periyalwar P, Dasarathy S. Malnutrition in cirrhosis: contribution and consequences of sarcopenia on metabolic and clinical responses. Clin Liver Dis. 2012;16(1):95-131. 